\title{
Downregulation of ST6GALNAC1 is associated with esophageal squamous cell carcinoma development
}

\author{
TAKESHI IWAYA ${ }^{1}$, GENTA SAWADA ${ }^{2}$, SUBURU AMANO ${ }^{1}$, KOHEI KUME ${ }^{1}$, CHIE ITO ${ }^{1}$, FUMITAKA ENDO ${ }^{1}$, \\ MASAFUMI KONOSU ${ }^{1}$, YOSHIHIRO SHIOI ${ }^{1}$, YUJI AKIYAMA ${ }^{1}$, TAKESHI TAKAHARA ${ }^{1}$, \\ KOKI OTSUKA ${ }^{1}$, HIROYUKI NITTA ${ }^{1}$, KEISUKE KOEDA ${ }^{1}$, MASARU MIZUNO ${ }^{1}$, \\ SATOSHI NISHIZUKA ${ }^{1}$, AKIRA SASAKI ${ }^{1}$ and KOSHI MIMORI ${ }^{3}$ \\ ${ }^{1}$ Department of Surgery, Iwate Medical University, Morioka, Iwate 020-8505; ${ }^{2}$ Department of Gastroenterological Surgery, \\ Graduate School of Medicine, Osaka, University, Suita 565-0871; ${ }^{3}$ Department of Surgery, \\ Kyushu University Beppu Hospital, Beppu 874-0838, Japan
}

Received October 20, 2016; Accepted December 16, 2016

DOI: $10.3892 /$ ijo.2016.3817

\begin{abstract}
Tylosis is an inherited disorder characterized by abnormal palmoplantar skin thickening and a highly elevated risk of esophageal squamous cell carcinoma (ESCC). Analyses of tylosis in families have localized the responsible gene locus to a region of chromosome 17q25.1. Frequent loss of heterozygosity ( $\mathrm{LOH}$ ) in 17q25.1 was also observed in the sporadic form of ESCC. A putative tumor suppressor gene for ESCC may exist at this locus. We investigated the expression patterns of genes on 17q25.1 in tumor and corresponding normal tissues from patients with sporadic ESCC using RNA sequence analysis. For candidate genes, quantitative real-time reverse transcription-PCR (qRT-PCR), direct sequence, $\mathrm{LOH}$ and methylation analyses were performed using 93 clinical ESCC samples and 10 cell lines. A significant downregulation of ST6GALNAC1 was demonstrated in ESCC tissues compared to its expression in normal tissues by qRT-PCR (n=93, p<0.0001). Frequent LOH $(17 / 27,62.9 \%)$ and hyper-methylation in ST6GALNAC1 were also observed in all cell lines. Our results indicated that ST6GALNAC1 was downregulated in sporadic ESCC via hyper-methylation and $\mathrm{LOH}$, and it may be a candidate responsible gene for ESCC. Furthermore, recent studies suggest that multiple genes on chromosome 17q25 are involved in ESCC development.
\end{abstract}

\section{Introduction}

Tylosis is an extremely rare autosomal, dominantly inherited disorder characterized by abnormal thickening of the palmoplantar skin and a highly elevated risk of esophageal squamous

Correspondence to: Professor Takeshi Iwaya, Department of Surgery, Iwate Medical University, 19-1, Uchimaru, Morioka, Iwate 020-8505, Japan

E-mail: takeiwaya@gmail.com

Key words: esophageal squamous cell carcinoma, ST6GALNAC1, hyper-methylation, chromosome 17q25, loss of heterozygosity, tumor suppressor gene cell carcinoma (ESCC). Five families with high frequencies of tylosis have been reported from the UK, USA, Germany, Spain and Finland (1-7). Linkage and haplotype analyses in these families have localized the tylosis esophageal cancer (TOC) gene locus to a region of chromosome $17 q 25(3-5,8,9)$. It has also been reported that frequent loss of heterozygosity $(\mathrm{LOH})$ in 17q25.1 was observed in the sporadic form of $\operatorname{ESCC}(10,11)$. These reports on inherited and sporadic ESCC indicated the presence of a putative tumor suppressor gene for ESCC at this locus. Although abnormalities of genes on 17q25.1, such as $C Y G B$ and $R H B D F 2$, have been demonstrated in families with tylosis, abnormalities of these genes have not been clearly demonstrated in sporadic forms of $\operatorname{ESCC}(12,13)$. Therefore, additional genes on $17 \mathrm{q} 25.1$ other than $C Y G B$ and $R H B D F 2$ may also be involved in ESCC development.

Additionally, we recently reported the result of whole-exome sequencing of paired DNA samples from 144 Japanese patients with ESCC (14). The most frequently mutated gene was TP53 (mutated in $93.1 \%$ of patients), followed by NOTCH1, MLL2, NFE2L2, ZNF750, FAT1 and PIK3CA (mutated in $10-20 \%$ of patients), in line with the results of next-generation sequencing analysis in other studies of sporadic ESCC (14-17). Although 17,189 non-synonymous mutations in 10,552 genes were identified in 144 ESCC samples in our previous study, recurrent mutations were not observed in genes on chromosome 17q25.1, including RHBDF2 (14). Other studies did not uncover RHBDF2 mutations either in sporadic ESCC $(16,17)$.

In this study, we investigated the expression patterns of genes in an $\sim 1500 \mathrm{~kb}$ region on $17 \mathrm{q} 25.1$, including the TOC locus, in tumor and corresponding normal tissues using RNA sequence (RNA-seq) analysis data from patients with sporadic ESCC. We demonstrated frequent downregulation of ST6 N-acetylgalactosaminide $\alpha$-2,6-sialyltransferase 1 (ST6GALNAC1) on 17q25.1 in ESCC tissues compared to its expression in corresponding normal tissues.

\section{Patients and methods}

Patients and sample collection. A total of 93 ESCC samples obtained by surgery were used after obtaining written 
Table I. Primer sequences and amplified regions of ST6GALNAC1 gene in direct sequencing analysis.

\begin{tabular}{lllc}
\hline Gene name & \multicolumn{1}{c}{ Forward primer } & \multicolumn{1}{c}{ Reverse primer } & Probe no. \\
\hline ST6GALNAC1 & CGAAATAGGAGGCCTTCAGA & AGAGAGTGAGGTTGGGCAGA & $\# 50$ \\
$E V P L$ & TACCGTGCCCTGTACGAGA & GCGCAGACCTGCTTCTGT & $\# 67$ \\
CYGB & CCGCTGCCTACAAGGAAGT & GGGTGGAGTTAGGGGTCCT & $\# 62$ \\
GAPDH & AGCCACATCGCTCAGACAC & GCCCAATACGACCAAATCC & $\# 60$ \\
\hline
\end{tabular}

Table II. Primer sequences and amplified regions of ST6GALNAC1 gene in direct sequencing analysis.

\begin{tabular}{llll}
\hline & \multicolumn{1}{c}{ Forward primer } & \multicolumn{1}{c}{ Reverse primer } & \multicolumn{1}{c}{ Amplified region } \\
\hline Segment 1 & CTTCCTTTAAGCCACGCCAGCTTAT & TGAAATATGAGGAGTGGAAAGGACA & 3'UTR and Exon 1 \\
Segment 2 & GCCTTCATCAAAGGTTATCTCTGTC & AAAGTCTAGAAGCAGAGCCCAGGAG & Exon 2 \\
Segment 3 & CCTTCCTGACTTCGTCCTCCTGTAT & GAGCTTGGGTGGGGACAGCTTAC & Exons 3 and 4 \\
Segment 4 & CCGTCTGATTGTGTCTTTCTGCAC & CAACCCTTTAGAGCCACTCATGACA & Exons 5 and 6 \\
Segment 5 & CCAGGTAAGGGAGCTGAGTCTGAAT & CCAGGAGCTGTTTCTCCAGGTATTT & Exons 7 and 8 \\
Segment 6 & CGTGATGTAGGTGAGTGCTTATGGC & TTCACTGTAGAAAATTTATTTGCCT & Exon 9 and 5'UTR \\
\hline
\end{tabular}

informed consent. All patients underwent resection of the primary tumor at Iwate Medical University, Kyushu University Beppu Hospital and affiliated hospitals between 1992 and 2007. Resected cancer and corresponding normal tissues were immediately cut and stored at $-80^{\circ} \mathrm{C}$ until DNA/RNA extraction. Total RNA and DNA were obtained using an RNeasy mini kit and QIAamp DNA mini kit (Qiagen Inc., Valencia, CA, USA), respectively.

Gene expression profiling on chromosome 17q25.1 using RNA-seq data of samples from three patients with ESCC. The expression patterns of genes on chromosome 17q25.1 were analyzed using RNA-seq data for tumor and corresponding normal tissues from three patients with ESCC. The characteristics of the three patients were as follows: well-differentiated SCC in the cervical esophagus (female, 70 years old, T1N0M0, stage I), poorly differentiated SCC in the middle thoracic esophagus (male, 73 years old, T3N0M0, stage II) and moderately differentiated SCC in the lower thoracic esophagus (male, 68 years old, T3N1M0, stage III). One microgram of extracted RNA was used as a template to construct RNA-seq libraries. Detailed protocols of RNA-seq analysis were described previously (18). Fold enrichment of the RNA-seq tags in the samples was calculated for each mRNA using the assigned tag counts and normalized to reads per kilobase $(\mathrm{kb}) \mathrm{mRNA}$.

Evaluation of ST6GALNAC1 and EVPL expression in clinical samples. Quantitative real-time reverse transcriptionPCR (qRT-PCR) was performed to measure ST6GALNAC1, EVPL, CYGB and glyceraldehyde 3-phosphate dehydrogenase $(G A P D H)$ mRNA expression using a LightCycler 480 Probes Master kit (Roche Applied Science, Mannheim, Germany) according to the manufacturer's protocol with specific primers and universal probes that were designed at the Universal Probe Library's assay design center (http://lifescience.roche. com/shop/CategoryDisplay?catalog $\mathrm{Id}=10001 \& \mathrm{t} \mathrm{ab}=$ Assay+Des
ign+Center\&identifier $=$ Universal+Probe+Libr ary\&langId=-1). Gene expression levels were normalized with respect to those of $G A P D H$. Primer sequences and universal probe number for each gene are listed in Table I.

Direct sequencing analysis of ST6GALNAC1. In 46 cases of ESCC, coding exons of ST6GALNACl were amplified using KOD FX (Toyobo, Tokyo, Japan) according to the manufacturer's protocol and sequenced using BigDye Terminator version 3.1 (Applied Biosystems, Foster City, CA, USA) as previously described (19). Primer sequences are listed in Table II and Fig. 1.

Microsatellite LOH analysis of the ST6GALNAC1 locus. In samples from 34 patients with ESCC, PCR was performed for three dinucleotide repeat microsatellite markers (D17S2238, D17S2243 and D17S2245) within the ST6GALNAC1 locus using fluorescent primer pairs (Applied Biosystems). LOH was analyzed using an ABI PRISM 3100 Genetic Analyzer and GeneScan Analysis and Genotyper software version 3.7.1 (Applied Biosystems).

Cell lines and cell culture. Ten human ESCC cell lines (KY150, KY270, KYSE410, KYSE450, KYSE510, TE1, TE6, TE8, TE9 and TE10) were purchased from the Japanese Collection of Research Bioresources Cell Bank and the Riken Bioresource Center. Cells were maintained in RPMI-1640 containing $10 \%$ fetal bovine serum and cultured in a humidified $5 \% \mathrm{CO}_{2}$ incubator at $37^{\circ} \mathrm{C}$.

Methylation levels and response to 5-aza-2'-deoxycytidine (5-Aza-dC) treatment in ESCC cell lines. ESCC cells were seeded at a density of $1 \times 10^{6}$ cells $/ 10 \mathrm{~cm}$ dish and cultured for $24 \mathrm{~h}$ with an inhibitor of DNA methyltransferase, 5-Aza-dC (Sigma-Aldrich, St. Louis, MO, USA), at a final concentration of $2 \mu \mathrm{M}$. Control cells were treated with the diluent 


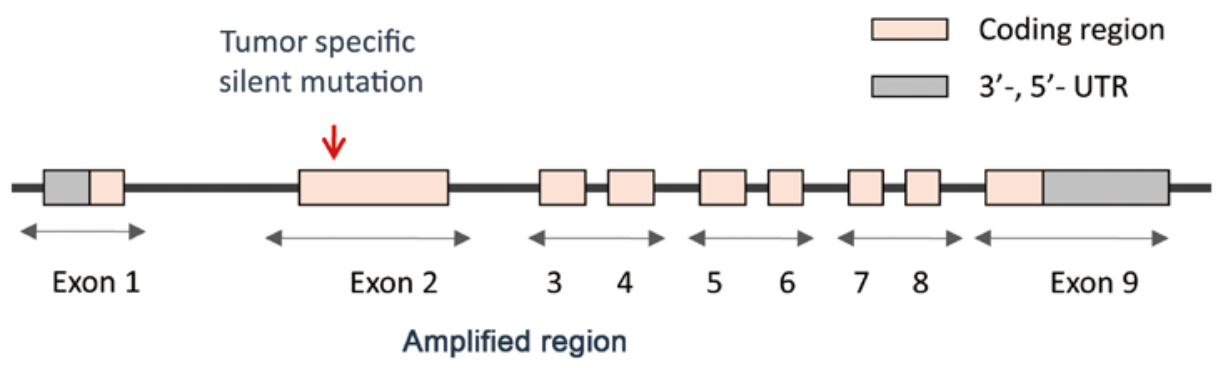

E152 c.509 G>A (p.Glu103Glu)
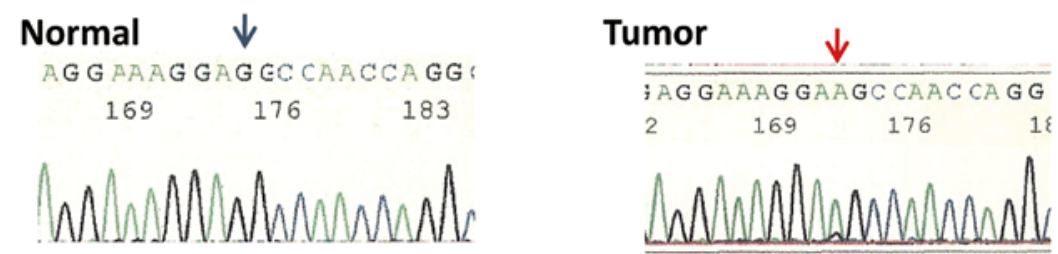

Figure 1. Sequence analysis of ST6GALNAC1. Sequence of ST6GALNAC1 is illustrated in the upper panel. All protein-coding regions were amplified by PCR. A tumor-specific silent mutation was noted in case E152.

phosphate-buffered saline (PBS) alone. After $48 \mathrm{~h}$ of incubation, total RNA was extracted from collected cells in each dish.

Statistical analysis. Data from RNA-seq analyses, qRT-PCR and methylation assays were analyzed using JMP 12 software (SAS Institute, Inc., Cary, NC, USA) and GraphPad Prism 6 (GraphPad Software Inc., La Jolla, CA, USA). Differences between the gene expression levels of samples were estimated using the Wilcoxon rank test or paired t-test. All differences were considered statistically significant at the level of $\mathrm{p}<0.05$.

\section{Results}

Differential gene expression profiling on chromosome 17q25.1 in ESCC samples by RNA-seq analysis. In our RNA-seq analysis from three patients with ESCC with differences in tumor stage, histological differentiation and tumor location, 17,673 genes were detected with an RPKM value of at least 2.0 in either normal or tumor tissues. The first 10 genes showed significant increase or decrease in their expression in tumor tissues compared with their expression in normal tissues and are listed in Table III. Among the expression data obtained by RNA-seq analysis, we focused on the expression profile of genes in a $1500 \mathrm{~kb}$ region on $17 \mathrm{q} 25.1$ including the TOC locus, which has been mapped to the $500 \mathrm{~kb}$ region in UK, USA and German pedigrees (8) and narrowed to a $42.5 \mathrm{~kb}$ region in the UK pedigree (9). The differences in expression levels between normal and tumor samples for 39 genes in the region are shown in Fig. 2. Among these genes, the expression levels of EVPL and ST6GALNAC1 in tumor tissues were significantly decreased to less than one-third of the levels in normal tissues (Fig. 2).

Downregulation of ST6GALNAC1 and EVPL in ESCC tissues. We validated the expression levels of these two genes using qRT-PCR in samples from 93 patients with ESCC. Both $E V P L$ and ST6GALNAC1 displayed significant downregulation in tumor samples compared to their corresponding normal tissue
Table III. Upregulated and downregulated genes in ESCC tumor by RNA-seq analysis.

\begin{tabular}{|c|c|c|c|}
\hline Genes & $\begin{array}{c}\text { Tumor } \\
\text { (average } \\
\text { RPKM) }\end{array}$ & $\begin{array}{l}\text { Normal } \\
\text { (average } \\
\text { RPKM) }\end{array}$ & $\begin{array}{c}\text { Fold-change } \\
\text { Tumor/ } \\
\text { normal }\end{array}$ \\
\hline \multicolumn{4}{|l|}{ Upregulated } \\
\hline CST1 & 160.41 & 0.21 & 763.84 \\
\hline$G R P$ & 6.88 & 0.01 & 516.25 \\
\hline$O B P 2 A$ & 7.77 & 0.02 & 466.4 \\
\hline$I L-17 C$ & 3.23 & 0.01 & 322.67 \\
\hline MMP11 & 160.47 & 0.64 & 250.74 \\
\hline RNASE10 & 5.27 & 0.03 & 175.56 \\
\hline$M M P 13$ & 2.44 & 0.02 & 146.6 \\
\hline$H I S T 1 H 3 G$ & 3.61 & 0.03 & 135.25 \\
\hline HOXD11 & 7.5 & 0.06 & 132.35 \\
\hline$M M P 3$ & 2.17 & 0.02 & 130 \\
\hline \multicolumn{4}{|l|}{ Downregulated } \\
\hline TMPRSS11B & 0.7 & 156.48 & 222.48 \\
\hline SFTA2 & 0.22 & 42.3 & 192.26 \\
\hline$M U C 21$ & 3.24 & 494.75 & 152.54 \\
\hline CRNN & 20.17 & 3015.85 & 149.5 \\
\hline KRT4 & 75.48 & 11106.92 & 147.15 \\
\hline$M A L$ & 21.72 & 2735.73 & 125.97 \\
\hline CWH43 & 0.21 & 15 & 70.3 \\
\hline KRT78 & 9.69 & 617.84 & 63.76 \\
\hline KRT13 & 610.37 & 37852.87 & 62.02 \\
\hline$C D 207$ & 0.06 & 3.79 & 59.89 \\
\hline
\end{tabular}

RPKM, read per kilobase per million mapped reads.

levels (p<0.0001) (Fig. 3). Although downregulation of $C Y G B$ has been demonstrated in esophageal tissues in tylotic patients 


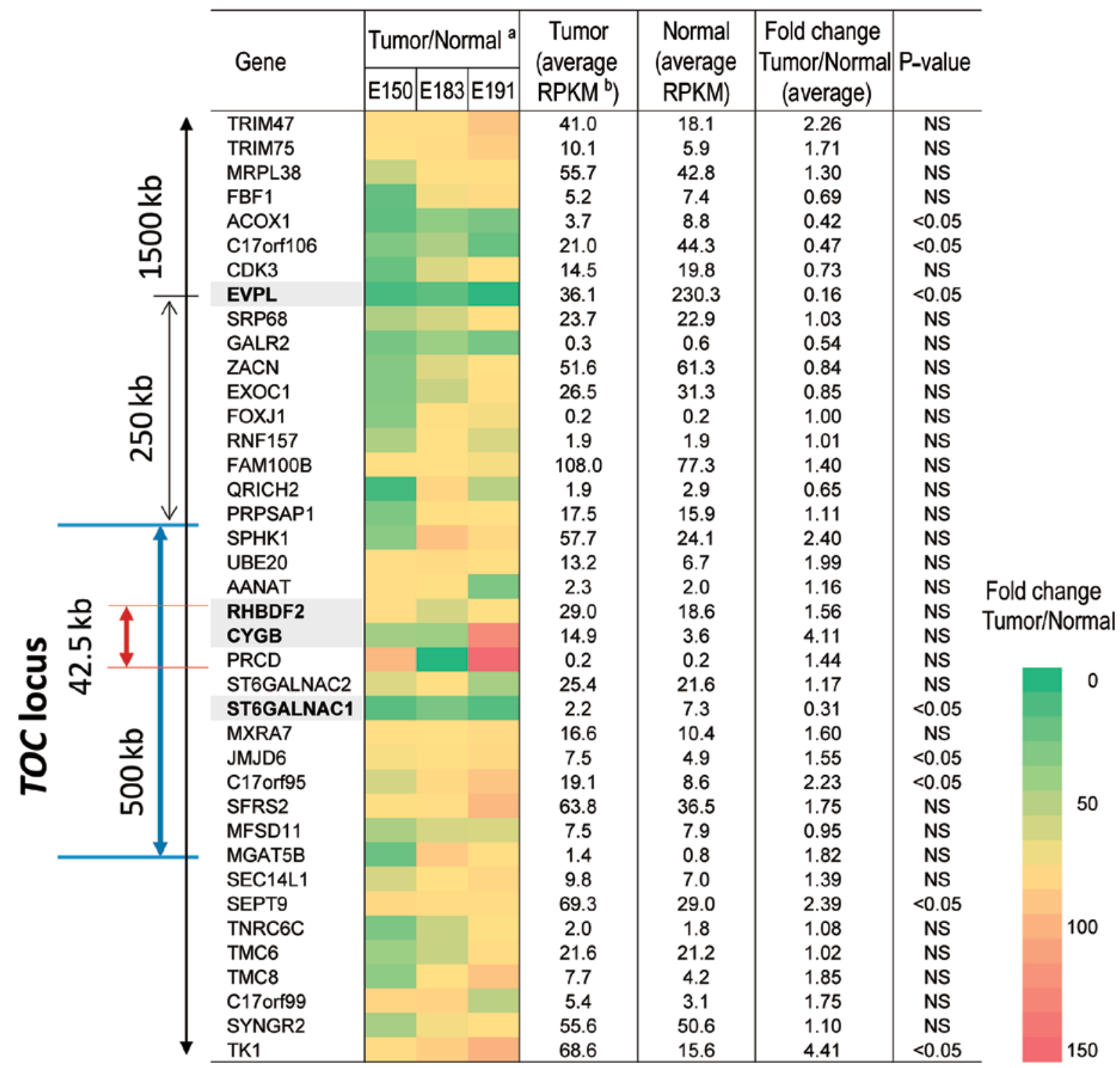

Figure 2. Gene expression patterns on chromosome 17q25.1 in esophageal squamous cell carcinoma (ESCC) samples. RPKMs of genes on 17q25.1 in tumor and corresponding normal tissues from three patients with ESCC are shown. a, Fold difference of RPKMs between tumor and normal tissues is schematized. The minimal region $(42.5 \mathrm{~kb})$ and $500 \mathrm{~kb}$ region of the TOC locus are shown on the left of the table. b, RPKMs, reads per kilobase per million mapped reads.
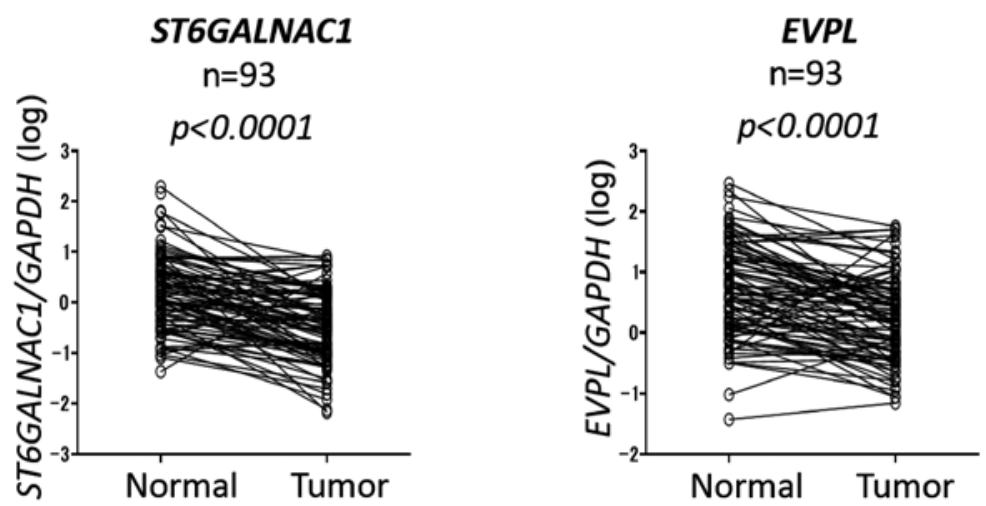

Figure 3. ST6GALNAC1 and EVPL expression in patients with esophageal squamous cell carcinoma (ESCC). ST6GALNAC1 and EVPL expression levels in tumor and corresponding normal tissues were validated by quantitative real-time reverse transcription-PCR (analysis in 93 patients with ESCC). The expression levels of both genes were normalized to those of $G A P D H$, and the results are shown as $\log _{10}$.

compared with that in the normal esophagus (12), a significant difference in its expression was not observed between sporadic
ESCC and normal samples by qRT-PCR analyses in our series (data not shown). 
A

ST6GALNAC1 gene

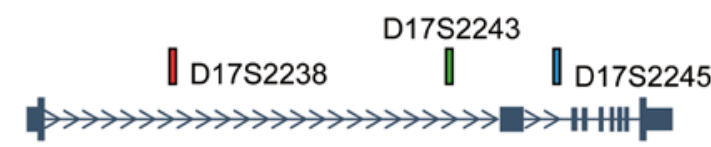

B

D17S2238

$\underline{D 17 S 2245}$

E150

E160

$\mathbf{N}$
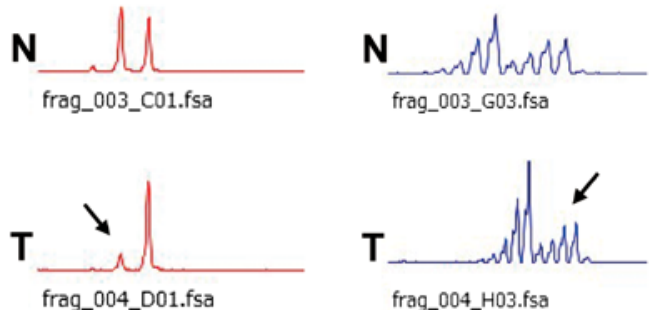

frag_003_G03.fsa

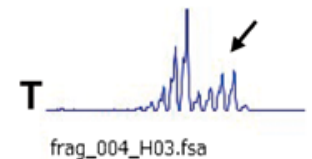

D17S2243

E155
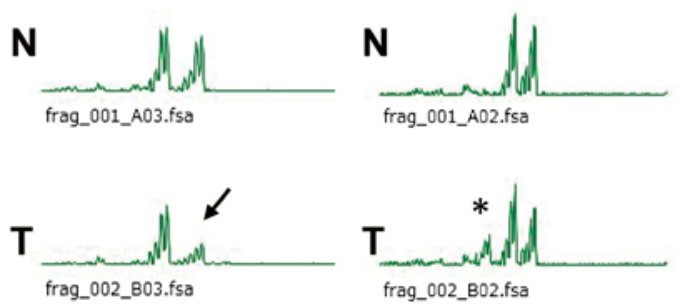

C

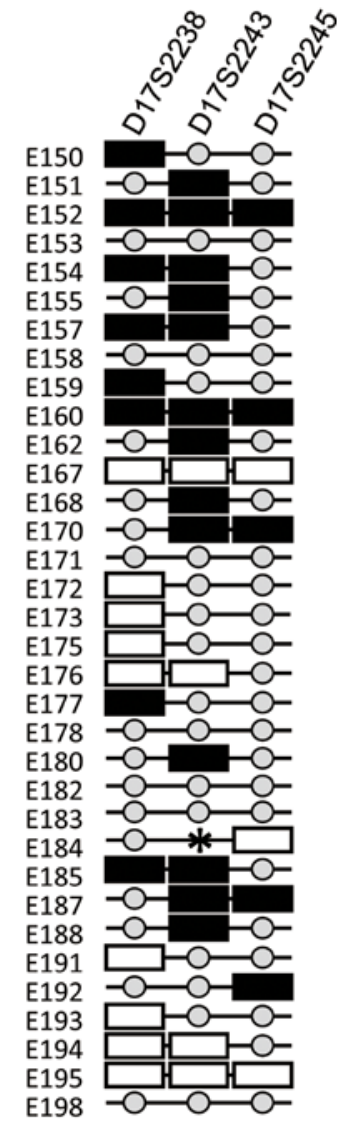

Homozygosity

Replication error

Figure 4. PCR microsatellite analysis at the ST6GALNAC1 locus in esophageal squamous cell carcinoma (ESCC) samples. (A) The locations of three microsatellite markers in the ST6GALNAC1 locus. D17S2238 and D17S2243 are located in intron 1 of ST6GALNAC1, and D17S2245 is located in intron 2. (B) Histograms of each marker in representative cases. Arrows indicate loss of heterozygosity (LOH), whereas the asterisk indicates a replication error. $\mathrm{N}$, normal; T, tumor. (C) LOH mapping on the ST6GALNACl locus. The microsatellite status, namely heterozygosity, homozygosity, $\mathrm{LOH}$ or replication error, at each marker in examined cases is represented schematically.

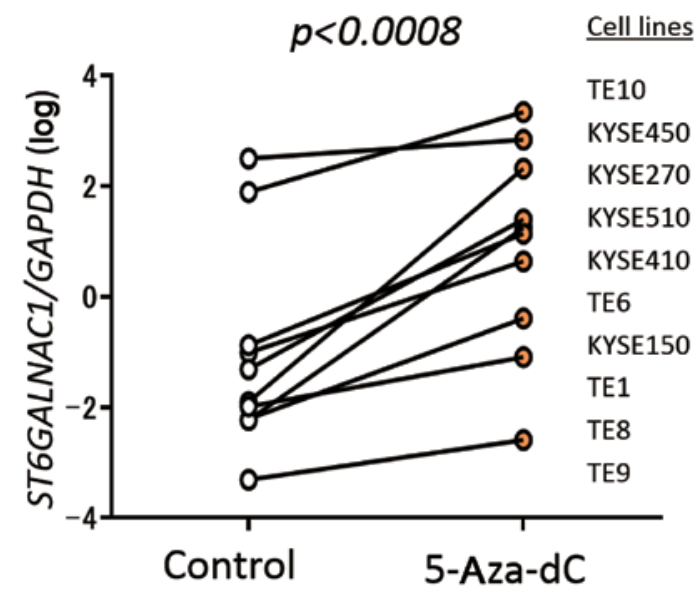

Figure 5. Methylation analysis of ST6GALNAC1 in esophageal squamous cell carcinoma (ESCC) cell lines. ESCC cell lines were treated with the DNA methyltransferase inhibitor 5-aza-2'-deoxycytidine (5-Aza-dC). ST6GALNAC1 expression levels were normalised to those of GAPDH, and the results are shown as $\log _{10}$.
Nucleotide variants in ST6GALNAC1. Direct sequence analyses of samples from 46 patients with ESCC revealed several nucleotide variants in ST6GALNAC1. Two missense variants [c.400 C>T (p.Pro67Leu) and c.724 C>T (p.Thr175Met)] were observed in two patients. In four patients, 3 bp in-frame deletions [c.752_754delTGG (p.His184del)] were observed. These variants were detected in both tumor and corresponding normal tissues, and they have been registered in dbSNP as rs143927446, rs138569950 and rs565363235. A one-base G insertion in intron 3 registered as rs146144287 in dbSNP was also detected in both tumor and normal tissues from six patients with ESCC. Only one patient $(1 / 46,2.1 \%)$ displayed a tumor-specific mutation in exon 2 of ST6GANACl, although this mutation was silent [c.509 G>A (p.Glu103Glu)] (Fig. 1). Therefore, no tumor-specific non-synonymous mutations were observed in the coding region of ST6GALNACl.

Frequent LOH was observed in the ST6GALNAC1 locus in ESCC samples. Three microsatellite markers, D17S2238, 
Table IV. Summary of altered genes on chromosome 17q25 in sporadic or hereditary forms of esophageal squamous cell carcinoma (ESCC).

\begin{tabular}{lrrll}
\hline Genes & Locus & $\begin{array}{c}\text { Position } \\
\text { (start-end) }\end{array}$ & \multicolumn{1}{c}{ Known function } & $\begin{array}{l}\text { Gene alterations in sporadic } \\
\text { ESCC or tylosis families }\end{array}$ \\
\hline EVPL & $17 \mathrm{q} 25.1$ & 76004502 & Link the cornified envelop to desmosomes & LOH and infrequent mutation \\
& & -76027452 & and intermediated filaments & Sporadic ESCC \\
RHBDF2 & $17 \mathrm{q} 25.1$ & 76471069 & Activation of the altered protein leading to constant & Germ-line missense mutations \\
& & -76501790 & EGF receptor signalling and hyper-proliferation & Tylosis families \\
CYGB & $17 \mathrm{q} 25.1$ & 76527356 & Collagen synthesis, O2 sensing and transport or & LOH and hyper-methylation \\
& & -76537905 & detoxification of reactive oxygen species & Tylosis families \\
ST6GALNAC1 & $17 \mathrm{q} 25.1$ & 76624763 & Synthesis of the sialyl-Tn antigen & LOH and hyper-methylation \\
& & -76643838 & in cancer cells & Sporadic ESCC \\
ZNF750 & $17 \mathrm{q} 25.3$ & 82828435 & Control terminal epidermal differentiation via & LOH and frequent mutation \\
& & -82840578 & interactions with KDM1A, RCOR1 and CTBP1/2 & Sporadic ESCC \\
\hline
\end{tabular}

D17S2243 and D17S2245, were located in the introns of ST6GALNAC1 (Fig. 4A). PCR microsatellite analysis of the ST6GALNAC1 locus in 34 patients using these three markers demonstrated LOH in 17/27 (62.9\%) informative cases at one or more sites and a replication error in $1 / 27(3.7 \%)$ cases at D17S2243 (Fig. 4B and C).

ST6GALNAC1 downregulation by methylation in ESCC cell lines. Treatment with 5-Aza-dC significantly elevated ST6GALNAC1 expression compared to the control level in all 10 ESCC cell lines assessed (Fig. 5).

\section{Discussion}

Because the region in chromosome $17 \mathrm{q} 25.1$ was affected in both hereditary and sporadic forms of ESCC, it is believed that a gene responsible for the oncogenesis or development of ESCC existed in this region. Several genes in the TOC locus have been studied in inherited and sporadic forms of ESCC. In our present study, ST6GALNACl expression was significantly decreased in ESCC tumor tissue compared with that in the corresponding normal tissue, and the gene was located in the vicinity of the minimal region of the TOC locus (Figs. 2 and 3). It was reported that ST6GALNACl was associated with biosynthesis of the sialyl-Tn (sTn) antigen in cancer cells and that it was overexpressed in gastric, breast and prostate cancer cell lines, inducing sTn expression (20). In contrast to these findings, our results suggest that ST6GALNAC1 may have a tumor-suppressor function in ESCC. In terms of the downregulation mechanisms of ST6GALNAC1 expression, frequent $\mathrm{LOH}(62.9 \%)$ of the gene locus was demonstrated in ESCC, although no tumor-specific mutation was observed in the coding region (Figs. 1 and 4). Furthermore, demethylation using 5-Aza-dC recovered ST6GALNAC1 expression in all 10 ESCC cell lines examined (Fig. 5). There are no CpG islands in the promoter region of ST6GALNACl. However, it has been reported that $S T 6 G A L N A C 1$ was downregulated by hypermethylation of GC 2 bp upstream of the transcription start site in estrogen receptor- and progesterone receptor-positive breast cancers (21). These results suggest that ST6GALNACl was inactivated by $\mathrm{LOH}$ and hyper-methylation of the transcription start site.

Several candidate genes for sporadic and inherited ESCC have been demonstrated. EVPL is a member of the desmosomal plaque protein family that attaches to desmosomal cadherin and keratin filaments. We previously reported infrequent mutations and frequent $\mathrm{LOH}$ of this gene in sporadic ESCC (22). In this study, we demonstrated that EVPL expression was significantly decreased in ESCC tissues (Fig. 3). Downregulation of EVPL may be involved in ESCC development, although the gene is located $250 \mathrm{~kb}$ to the telomeric side of the minimal region of the TOC locus (Fig. 2). It has been demonstrated that $C Y G B$ was a tumor suppressor gene inactivated by DNA hyper-methylation of its promoter in several types of cancer, including ESCC $(12,23)$. However, downregulation of $C Y G B$ was not observed in our series of tumor tissues from Japanese patients with ESCC compared to its levels in the corresponding normal tissues (data not shown). CYGB methylation has also been demonstrated in multiple malignancies other than ESCC, such as leukaemia as well as breast, bladder, lung and colon cancers (23). These findings indicated that alterations of $C Y G B$ were limited to a subset of ESCCs and that the tumor-suppressor role of $C Y G B$ may not be specific in esophageal tissues but instead may be common among many types of malignancies.

Recently, missense mutations of $R H B D F 2$ in the minimal region of the TOC locus were identified in patients with tylosis from US/UK, German and Finnish families $(7,13)$. It may be clear that $R H B D F 2$ is a responsible gene for tylosis. Blaydon et al demonstrated that the altered $R H B D F 2$ represents a gain-of-function allele that results in sustained EGFR signaling within the cells, and the signaling leads to a hyperproliferative phenotype. Furthermore, it was suggested that RHBDF2 may also be dysregulated in a similar manner in sporadic ESCC according to immunohistochemical data (13). In our previous study, however, RHBDF 2 mutation was not observed by whole-exome sequence analysis using next-generation sequencing in 144 patients with sporadic ESCC (14). This sequencing analysis also demonstrated that recurrent mutations were observed only in ZNF750, with the mutation 
rate of $16.7 \%$, on chromosome 17q. Frequent $Z N F 750$ mutations were also demonstrated in Chinese patients with sporadic ESCC by whole-exome sequence analysis (15). Furthermore, we found that the mutational APOBEC signature was predominantly observed in the ESCC genome, and ZNF750 mutations were positively associated with the APOBEC signature (14). ZNF750 was located on 17q25.3 telomeric to the TOC locus, and its mutations were null mutations accompanied by LOH (14). Therefore, ZNF750 may be a strong candidate tumor suppressor gene for ESCC. It remains unclear as to which gene dysregulation of in the chromosomal region is essential for the development of hereditary and sporadic forms of ESCC. Mutations in one allele of $R H B D F 2$ gene induced sustained EGFR signaling in the cells and led to a hyperproliferative phenotype during wound repair in patients with tylosis (13). Although RHBDF2 mutation was not observed in sporadic ESCC, the EGFR signaling were frequently dysregulated in sporadic ESCC cells (14-17). Therefore, an abnormality in the RHBDF2-EGFR pathway may lead to precancerous lesions in the esophagus. In addition to the oncogenic change in RHBDF2-EGFR, further inactivation of several tumor suppressor genes on 17q25, such as EVPL, CYGB and ZNF750, by a two-hit mechanism may induce ESCC.

In conclusion, ST6GALNAC1 was downregulated in sporadic ESCC by hyper-methylation and $\mathrm{LOH}$, and it may be a candidate responsible gene for ESCC. Furthermore, our results on sporadic ESCC and recent studies on tylotic families suggest that multiple genes on chromosome 17q25 are involved in ESCC development (Table IV).

\section{Acknowledgements}

This study was supported by JSPS KAKENHI (grant nos. JP23591937 and JP26461994).

\section{References}

1. Ellis A, Field JK, Field EA, Friedmann PS, Fryer A, Howard P, Leigh IM, Risk J, Shaw JM and Whittaker J: Tylosis associated with carcinoma of the oesophagus and oral leukoplakia in a large Liverpool family - a review of six generations. Eur J Cancer B Oral Oncol 30B: 102-112, 1994.

2. Stevens HP, Kelsell DP, Bryant SP, Bishop DT, Spurr NK, Weissenbach J, Marger D, Marger RS and Leigh IM: Linkage of an American pedigree with palmoplantar keratoderma and malignancy (palmoplantar ectodermal dysplasia type III) to 17q24. Literature survey and proposed updated classification of the keratodermas. Arch Dermatol 132: 640-651, 1996.

3. Hennies HC, Hagedorn M and Reis A: Palmoplantar keratoderma in association with carcinoma of the esophagus maps to chromosome $17 \mathrm{q}$ distal to the keratin gene cluster. Genomics 29 : 537-540, 1995.

4. Kelsell DP, Risk JM, Leigh IM, Stevens HP, Ellis A, Hennies HC, Reis A, Weissenbach J, Bishop DT, Spurr NK, et al: Close mapping of the focal non-epidermolytic palmoplantar keratoderma (PPK) locus associated with oesophageal cancer (TOC). Hum Mol Genet 5: 857-860, 1996.

5. Risk JM, Field EA, Field JK, Whittaker J, Fryer A, Ellis A, Shaw JM, Friedmann PS, Bishop DT, Bodmer J, et al: Tylosis oesophageal cancer mapped. Nat Genet 8: 319-321, 1994.

6. Varela AB, Blanco Rodríguez MM, Boullosa PE and Silva JG: Tylosis A with squamous cell carcinoma of the oesophagus in a Spanish family. Eur J Gastroenterol Hepatol 23: 286-288, 2011.
7. Saarinen S, Vahteristo P, Lehtonen R, Aittomäki K, Launonen V, Kiviluoto T and Aaltonen LA: Analysis of a Finnish family confirms RHBDF2 mutations as the underlying factor in tylosis with esophageal cancer. Fam Cancer 11: 525-528, 2012.

8. Risk JM, Evans KE, Jones J, Langan JE, Rowbottom L, McRonald FE, Mills HS, Ellis A, Shaw JM, Leigh IM, et al: Characterization of a $500 \mathrm{~kb}$ region on $17 \mathrm{q} 25$ and the exclusion of candidate genes as the familial tylosis oesophageal cancer (TOC) locus. Oncogene 21: 6395-6402, 2002.

9. Langan JE, Cole CG, Huckle EJ, Byrne S, McRonald FE, Rowbottom L, Ellis A, Shaw JM, Leigh IM, Kelsell DP, et al: Novel microsatellite markers and single nucleotide polymorphisms refine the tylosis with oesophageal cancer (TOC) minimal region on $17 q 25$ to $42.5 \mathrm{~kb}$ : Sequencing does not identify the causative gene. Hum Genet 114: 534-540, 2004.

10. Iwaya T, Maesawa C, Ogasawara S and Tamura G: Tylosis esophageal cancer locus on chromosome $17 \mathrm{q} 25.1$ is commonly deleted in sporadic human esophageal cancer. Gastroenterology 114: 1206-1210, 1998

11. von Brevern M, Hollstein MC, Risk JM, Garde J, Bennett WP, Harris CC, Muehlbauer KR and Field JK: Loss of heterozygosity in sporadic oesophageal tumors in the tylosis oesophageal cancer (TOC) gene region of chromosome 17q. Oncogene 17: 2101-2105, 1998.

12. McRonald FE, Liloglou T, Xinarianos G, Hill L, Rowbottom L, Langan JE, Ellis A, Shaw JM, Field JK and Risk JM: Downregulation of the cytoglobin gene, located on $17 \mathrm{q} 25$, in tylosis with oesophageal cancer (TOC): Evidence for trans-allele repression. Hum Mol Genet 15: 1271-1277, 2006.

13. Blaydon DC, Etheridge SL, Risk JM, Hennies HC, Gay LJ, Carroll R, Plagnol V, McRonald FE, Stevens HP, Spurr NK, et al: RHBDF2 mutations are associated with tylosis, a familial esophageal cancer syndrome. Am J Hum Genet 90: 340-346, 2012.

14. Sawada G, Niida A, Uchi R, Hirata H, Shimamura T, Suzuki Y, Shiraishi Y, Chiba K, Imoto S, Takahashi Y, et al: Genomic landscape of esophageal squamous cell carcinoma in a Japanese population. Gastroenterology 150: 1171-1182, 2016.

15. Lin DC, Hao JJ, Nagata Y, Xu L, Shang L, Meng X, Sato Y, Okuno Y, Varela AM, Ding LW, et al: Genomic and molecular characterization of esophageal squamous cell carcinoma. Nat Genet 46: 467-473, 2014.

16. Song Y, Li L, Ou Y, Gao Z, Li E, Li X, Zhang W, Wang J, Xu L, Zhou Y, et al: Identification of genomic alterations in oesophageal squamous cell cancer. Nature 509: 91-95, 2014.

17. Gao YB, Chen ZL, Li JG, Hu XD, Shi XJ, Sun ZM, Zhang F, Zhao ZR, Li ZT, Liu ZY, et al: Genetic landscape of esophageal squamous cell carcinoma. Nat Genet 46: 1097-1102, 2014.

18. Iwaya T, Fukagawa T, Suzuki Y, Takahashi Y, Sawada G, Ishibashi M, Kurashige J, Sudo T, Tanaka F, Shibata K, et al: Contrasting expression patterns of histone mRNA and microRNA 760 in patients with gastric cancer. Clin Cancer Res 19: 6438-6449, 2013.

19. Yokobori T, Mimori K, Iwatsuki M, Ishii H, Onoyama I, Fukagawa T, Kuwano H, Nakayama KI and Mori M: p53-altered FBXW7 expression determines poor prognosis in gastric cancer cases. Cancer Res 69: 3788-3794, 2009.

20. Munkley J: The role of Sialyl-Tn in cancer. Int J Mol Sci 17: 275, 2016.

21. Li L, Lee KM, Han W, Choi JY, Lee JY, Kang GH, Park SK, Noh DY, Yoo KY and Kang D: Estrogen and progesterone receptor status affect genome-wide DNA methylation profile in breast cancer. Hum Mol Genet 19: 4273-4277, 2010.

22. Iwaya T, Maesawa C, Kimura T, Ogasawara S, Ikeda K, Kimura Y, Noda Y, Ishida K, Sato N, Saito K, et al: Infrequent mutation of the human envoplakin gene is closely linked to the tylosis oesophageal cancer locus in sporadic oesophageal squamous cell carcinomas. Oncol Rep 13: 703-707, 2005.

23. Shivapurkar N, Stastny V, Okumura N, Girard L, Xie Y, Prinsen C, Thunnissen FB, Wistuba II, Czerniak B, Frenkel E, et al: Cytoglobin, the newest member of the globin family, functions as a tumor suppressor gene. Cancer Res 68: 7448-7456, 2008. 